\title{
Gravitational collapse of a Hagedorn fluid in Vaidya geometry
}

\author{
T. Harko* \\ Department of Physics, The University of Hong Kong, Pokfulam Road, Hong Kong, People's Republic of China
}

(Received 22 April 2003; published 9 September 2003)

\begin{abstract}
The gravitational collapse of a high-density null charged matter fluid, satisfying the Hagedorn equation of state, is considered in the framework of the Vaidya geometry. The general solution of the gravitational field equations can be obtained in an exact parametric form. The conditions for the formation of a naked singularity, as a result of the collapse of the compact object, are also investigated. For an appropriate choice of the arbitrary integration functions the null radial outgoing geodesic, originating from the shell focussing central singularity, admits one or more positive roots. Hence a collapsing Hagedorn fluid could end either as a black hole, or as a naked singularity. A possible astrophysical application of the model, to describe the energy source of gammaray bursts, is also considered.
\end{abstract}

DOI: 10.1103/PhysRevD.68.064005

PACS number(s): 04.20.Cv, 04.20.Dw, 04.20.Jb

\section{INTRODUCTION}

Investigating the final fate of the gravitational collapse of an initially regular distribution of matter, in the framework of the Einstein theory of gravitation, is one of the most active fields of research in contemporary general relativity. One would like to know whether, and under what initial conditions, gravitational collapse results in black hole formation. One would also like to know if there are physical collapse solutions that lead to naked singularities. If found, such solutions would be counterexamples of the cosmic censorship hypothesis, which states that curvature singularities in asymptotically flat space-times are always shrouded by event horizons.

Penrose [1] was the first to propose the idea, known as the cosmic conjecture: does a cosmic censor exist who forbids the occurrence of naked singularities, clothing each one in an absolute event horizon? This conjecture can be formulated in a strong sense (in a reasonable space-time we cannot have a naked singularity) or in a weak sense (even if such singularities occur they are safely hidden behind an event horizon, and therefore cannot communicate with far-away observers). Since Penrose's proposal, there have been various attempts to prove this conjecture (see [2] and references therein). Unfortunately, none have been successful so far.

Since, due to the complexity of the full Einstein equations, the general problem appears intractable, metrics with special symmetries are used to construct gravitational collapse models. One such case is the two-dimensional reduction of general relativity obtained by imposing spherical symmetry. Even with this reduction, however, very few inhomogeneous exact nonstatic solutions have been found. One well-known example is the Vaidya metric [3]. It describes the gravitational field associated with the eikonal approximation of an isotropic flow of unpolarized radiation, or, in other words, it represents a null fluid. It is asymptotically flat and it is employed in modeling the external field of radiating stars and evaporating black holes. The second one is the Tolman-Bondi metric [4], which gives the gravitational

*Email address: harko@hkucc.hku.hk field associated with dust matter and is frequently applied either in cosmological models or in describing the collapse of a star to a black hole. Tolman-Bondi space-times embody the Schwarzschild solution, the Friedman universes, and the Oppenheimer-Snyder collapse, as well as inhomogeneous expansions and collapses.

At first sight these two metrics are completely different. Do the naked singularities that form in the collapse of null radiation and in the collapse of dust bear any relation with each other? Are there any features common to both solutions? And if this is the case, what are the implications for cosmic censorship? As shown by Lemos [5], the naked singularities which appear in Vaidya and Tolman-Bondi spacetimes are of the same nature. Various important features such as the degree of inhomogeneity of the collapse necessary to produce a naked singularity, the Cauchy horizon equation, the apparent horizon equation, the strength of the singularity and the stability of the space-time have a mutual correspondence in both metrics. For cosmic censorship, this result implies that if the shell-focusing singularities arising from the collapse of a null fluid are not artifacts of some (eikonal) approximation, then the shell-focusing singularities arising from the collapse of dust are also not artifacts (and vice versa). Conversely, if the naked singularities are artifacts in one of them so are they in the other.

Thus the Vaidya metric belongs to the Tolman-Bondi family. The most unbound case yields the Vaidya metric. Hence one expects that major features which might arise in one of the metrics will also appear in the other. One example is the result that the strength in the Vaidya metric depends on the direction from which the geodesics enter the singularity [5].

Null fluids are, in principle, easier to treat than matter fields. A null fluid is the eikonal approximation of a massless scalar field. Thus if one shows that the naked singularities arising in the Vaidya metric can be derived from more fundamental (massless) fields, then the naked singularities, which form in the Tolman-Bondi collapse, may also be derived from more fundamental (massive) fields. The same types of relations and conclusions hold for charged radiation and charged dust matter. The structure and properties of singularities in the gravitational collapse in Vaidya space-times have been analyzed, from different points of view, in [6-10]. 
Within the framework of various physical models, the spherical gravitational collapse has been analyzed in many papers. The role of initial density and velocity distributions towards determining the final outcome of spherical dust collapse and the causal structure of the singularity has been examined in terms of evolution of apparent horizon in $[11,12]$. The collapse was described by the Tolman-Bondi metric with two free functions. The collapse can end in either a black hole or a naked singularity. The occurrence and nature of naked singularities in the Szekeres space-times have been investigated in [13]. These space-times represent irrotational dust. They do not have any Killing vectors and they are the generalizations of the Tolman-Bondi space-times. There also exist naked singularities that satisfy both the limiting focusing condition and the strong limiting focusing conditions. The relevance of the initial state of a collapsing dust cloud towards determining its final fate in the course of a continuing gravitational collapse has been considered in [14]. Given any arbitrary matter distribution for the cloud in the initial epoch, there is always the freedom to chose the rest of the initial data, namely the initial velocities of the collapsing spherical shells, so that, depending on this choice, the collapse could result either in a black hole or a naked singularity. Thus, given the initial density profile, to achieve the desired end state of the gravitational collapse one has to give a suitable initial velocity of the cloud. The expression for the expansion of outgoing null geodesics in spherical dust collapse has been derived in [15]. The limiting values of the expansion in the approach to singularity formation have been computed. Using these results one can show that the covered, as well as the naked, singularity solutions arising in spherical dust collapse, are stable under small changes in the equation of state.

The growth of the Weyl curvature is examined in two examples of naked singularity formation in spherical gravitational collapse dust and Vaidya space-time in [16]. The Weyl scalar diverges along outgoing radial null geodesics as they meet the naked singularity in the past. Although general relativity admits naked singularities arising from gravitational collapse, the second law of thermodynamics could forbid their occurrence in nature. A simple model for a corona of a neutrino-radiating star showing critical behavior is presented in [17]. The conditions for the existence or absence of a bounce (explosion) are discussed. The charged Vaidya metric was extended to cover all of the space-time in [18] and the Penrose diagram for the formation and evaporation of a charged black hole obtained. The covariant equations characterizing the strength of a singularity in spherical symmetry and a slight modification to the definition of singularity strength have been derived in [19]. The idea of probing naked space-times singularities with waves rather than with particles has been proposed in [20]. For some space-times the classical singularity becomes regular if probed with waves, while stronger classical singularities remain singular.

In order to obtain the energy-momentum tensor for the collapse of a null fluid an inverted approach was proposed by Husain [21]. First the stress-energy momentum tensor is determined from the metric. Then the equation of state and the dominant energy condition are imposed on its eigenvalues.
This leads to an equation for the metric function. The precise form of the stress-energy tensor is then displayed. By using this approach several classes of solutions describing the collapse of a null fluid, satisfying barotropic and polytropic type equations of state, have been obtained. In the framework of the same approach a large class of solutions, including type-II fluids, and which includes most of the known solutions of the Einstein field equations, has been derived, in four dimensions by Wang and $\mathrm{Wu}$ [22], and in $N \geqslant 4$ dimensions by Villas da Rocha [23]. The Vaidya radiating metric has been extended to include both a radiation field and a string fluid by Glass and Krisch [24,25] and by Govinder and Govender [26].

When nuclear matter is squeezed to a sufficiently high density, a phase transition takes place and neutron matter converts into three-flavor (strange) quark matter, which is due to the fact that strange matter may be more stable than nuclear matter. The collapse of the quark fluid, described by the bag model equation of state $p=(\rho-4 B) / 3$, with $B$ $=$ const, has been studied by Harko and Cheng [27] and the conditions for the formation of a naked singularity have been obtained. The obtained solution has been generalized to arbitrary space-time dimensions and to a more general linear equation of state by Ghosh and Dadhich $[28,29]$.

In 1965 Hagedorn [30] postulated that for large masses $m$ the spectrum of hadrons $\rho(m)$ grows exponentially, $\rho(m)$ $\sim \exp \left(m / T_{H}\right)$, where $T_{H}$, the Hagedorn temperature, is a scale parameter. The hypothesis was based on the observation that at some point a further increase of energy in protonproton and proton-antiproton collisions no longer raises the temperature of the formed fireball, but results in more and more particles being produced. Thus there is a maximum temperature $T_{H}$ that a hadronic system can achieve. The statistical model of the hadrons has been used to obtain a description of dense matter at densities exceeding nuclear density. The Hagedorn phase also arises in theories containing fundamental strings, because they have a large number of internal degrees of freedom [31]. As a result of the existence of many oscillator modes the density of states grows exponentially with single string energy. Thermodynamical quantities, such as the entropy, diverge at the Hagedorn temperature. If one considers an ensemble of weekly interacting strings at finite temperature, this behavior of the density of states is thought to lead either to a limiting temperature or a phase transition, in which the string configuration changes to one which is dominated by a single long string [32]. The high density Hagedorn phase of matter has been extensively used in cosmology to describe the very early phases of the evolution of the Universe [33-36].

It is the purpose of the present paper to study the spherically symmetric gravitational collapse of the charged matter in the Hagedorn phase. In order to simplify the mathematical formalism we adopt the assumption that the high density fluid moves along the null geodesics of a Vaidya type spacetime. The Vaidya geometry, also permitting the incorporation of the effects of the radiation, offers a more realistic background than static geometries, where all back reaction is ignored. By adopting the Hagedorn equation of state for dense matter, the general solution of the field equations can be 
obtained in an exact form. For the sake of comparison we also consider the collapse of matter described by the stiff Zeldovich equation of state. The conditions of formation of naked singularities are obtained in both cases.

The present paper is organized as follows. The field equations describing the collapse of a Hagedorn fluid are written down in Sec. II. The general solution of the field equations is presented in Sec. III. The equation of the null outgoing geodesics and the conditions of the formation of the naked singularities are discussed in Sec. IV. An astrophysical application of the formalism to explain the gamma ray bursts energy emissions is described in Sec. V. In Sec. VI we discuss and conclude our results.

\section{GEOMETRY AND FIELD EQUATIONS}

In ingoing Bondi coordinates $(u, r, \theta, \varphi)$ and with advanced Eddington time coordinate $u=t+r$ (with $r \geqslant 0$ the radial coordinate and $r$ decreasing towards the future) the line element describing the radial collapse of a coherent stream of matter can be represented in the form $[21,27]$

$$
d s^{2}=-\left[1-\frac{2 m(u, r)}{r}\right] d u^{2}+2 d u d r+r^{2}\left(d \theta^{2}+\sin ^{2} \theta d \varphi^{2}\right) .
$$

$m(u, r)$ is the mass function and gives the gravitational mass within a given radius $r$. In the following we use the natural system of units with $8 \pi G=c=1$.

The matter energy-momentum tensor can be written in the form $[21,22]$

$$
T_{\mu \nu}=T_{\mu \nu}^{(n)}+T_{\mu \nu}^{(m)}+E_{\mu \nu},
$$

where

$$
T_{\mu \nu}^{(n)}=\mu(u, r) l_{\mu} l_{\nu}
$$

is the component of the matter field that moves along the null hypersurfaces $u=$ const,

$$
T_{\mu \nu}^{(m)}=(\rho+p)\left(l_{\mu} n_{\nu}+l_{\nu} n_{\mu}\right)+p g_{\mu \nu}
$$

represents the energy-momentum tensor of the collapsing matter, and

$$
E_{\mu \nu}=\frac{1}{4 \pi}\left(F_{\mu \alpha} F_{\nu}^{\alpha}-\frac{1}{4} g_{\mu \nu} F_{\alpha \beta} F^{\alpha \beta}\right)
$$

is the electromagnetic contribution. $l_{\mu}$ and $n_{\mu}$ are two null vectors given by $l_{\mu}=\delta_{(\mu)}^{(0)}$ and $n_{\mu}=\frac{1}{2}\{1-[2 m(u, r) / r]\} \delta_{(\mu)}^{(0)}$ $-\delta_{(\mu)}^{(1)}$, so that $l_{\alpha} l^{\alpha}=n_{\alpha} n^{\alpha}=0$ and $l_{\alpha} n^{\alpha}=-1$ (with $\delta_{(b)}^{(a)}$ the Kronecker symbol) [21,22]. The energy density and pressure in Eq. (4) have been obtained by diagonalizing the energymomentum tensor obtained from the metric [21].

The electromagnetic tensor $F_{\mu \nu}$ obeys the Maxwell equations [37]

$$
\frac{\partial F_{\mu \nu}}{\partial x^{\lambda}}+\frac{\partial F_{\lambda \mu}}{\partial x^{\nu}}+\frac{\partial F_{\nu \lambda}}{\partial x^{\mu}}=0,
$$

$$
\frac{1}{\sqrt{-g}} \frac{\partial}{\partial x^{\mu}}\left(\sqrt{-g} F^{\mu \nu}\right)=-4 \pi j^{\mu} .
$$

Without any loss of generality the electromagnetic vector potential can be chosen as $[6,7]$

$$
A_{\mu}=\frac{q(u)}{r} \delta_{(\mu)}^{(u)},
$$

with $q(u)$ being an arbitrary integration function. From the Maxwell equations (6) and (7), it follows that the only nonvanishing components of $F_{\mu \nu}$ are $F_{r u}=-F_{u r}=q(u) / r^{2}$ and, consequently,

$$
E_{\mu}^{\nu}=\frac{q^{2}(u)}{r^{4}} \operatorname{diag}(-1,1,-1,1) .
$$

For the energy-momentum tensor (2) the gravitational field equations take the form [27]

$$
\begin{aligned}
\frac{1}{r^{2}} \frac{\partial m(u, r)}{\partial u} & =\frac{1}{2} \mu(u, r), \\
\frac{2}{r^{2}} \frac{\partial m(u, r)}{\partial r} & =\rho(u, r)+\frac{q^{2}(u)}{r^{4}}, \\
-\frac{1}{r} & \frac{\partial^{2} m(u, r)}{\partial r^{2}}=p(u, r)+\frac{q^{2}(u)}{r^{4}} .
\end{aligned}
$$

The stress-energy tensor (4) satisfies the dominant energy condition if the following three conditions are met:

$$
p \geqslant 0, \quad \rho \geqslant p, \quad T_{a b} w^{a} w^{b}>0,
$$

where $w^{a}$ is an arbitrary timelike four-vector. The first two of these conditions imply that $\partial m / \partial r \geqslant 0$ and $\partial^{2} m / \partial r^{2} \leqslant 0$. The former just says that the mass function either increases with $r$ or is a constant, which is a natural physical requirement on it. To satisfy the first two of the dominant energy conditions one must impose an equation of state for the collapsing matter.

Usually two different equations of state are used for the description of matter at extremely high densities. One of the most widely investigated cases is the so-called causal limit of the linear barotropic equation of state $p=(\gamma-1) \rho, \gamma$ $=$ const, corresponding to $\gamma=2$, or the Zeldovich stiff fluid equation of state $p=\rho$.

The Zeldovich equation of state, valid for densities significantly higher than nuclear densities, $\rho>10 \rho_{n u c}$, with $\rho_{\text {nuc }}=10^{14} \mathrm{~g} / \mathrm{cm}^{3}$, can be obtained by constructing a relativistic Lagrangian that allows bare nucleons to interact attractively via scalar meson exchange and repulsively via the exchange of a more massive vector meson [38]. In the nonrelativistic limit both the quantum and classical theories yield Yukawa-type potentials. At the highest densities the vector meson exchange dominates and by using a mean field approximation one can show that in the extreme limit of 
infinite densities the pressure tends to the energy density, $p$ $\rightarrow \rho$. In this limit the sound speed $c_{s}=\sqrt{d p / d \rho} \rightarrow 1$, and hence this equation of state satisfies the causality condition, with the speed of sound less than the speed of light [38].

An alternative approach to the equation of state at ultrahigh densities is based on the assumption that a whole host of baryonic resonant states arise at high densities. In the Hagedorn model the baryon resonance mass spectrum, that is the increase in the number of species of particles with mass between $m$ and $m+d m$, is given by $d N=N(m) d m$ $\sim a \exp \left(m / T_{H}\right) /\left(m_{0}^{2}+m^{2}\right)^{l} d m$, where $N(m) d m$ is the number of resonances between mass $m$ and $m+d m$. Fitting to the existent experimental data on baryon resonances show that $m_{0}=500 \mathrm{MeV}$ and $a=2.63 \times 10^{4} \mathrm{MeV}^{3 / 2}$ [39]. If $d N$ increased any faster as $m \rightarrow \infty$ than in the above formula, the partition function would not converge. Also the partition function converges only if the temperature of the system is less than $T_{H}$. Thus $T_{H}$, the Hagedorn temperature, is the effective highest temperature for any system. By interpreting the transverse momentum distribution of secondaries in very high energy collisions in terms of the model one obtains $T_{H} \sim 150-190 \mathrm{MeV}$ and $l=5 / 4$ [39]. The corresponding equation of state for the matter is

$$
p=p_{0}+\rho_{0} \ln \frac{\rho}{\rho_{0}}
$$

where $p_{0}=0.314 \times 10^{14} \mathrm{~g} / \mathrm{cm}^{3}$ and $\rho_{0}=1.253 \times 10^{14} \mathrm{~g} / \mathrm{cm}^{3}$ $[40,41]$. The velocity of sound in this type of matter is $c_{s}$ $=\sqrt{\rho_{0} / \rho}$. For the Hagedorn equation of state the speed of sound has the property $c_{s} \rightarrow 0$ for $\rho / \rho_{0} \rightarrow \infty$, in striking contrast with the mean field theory approach, leading to the Zeldovich equation of state, in which $c_{s} \rightarrow 1$. The Hagedorn equation of state creates new "particles" continuously with increasing density, rather than enlarging the Fermi sea of a single species. The equation of state (14) could be valid asymptotically for densities greater than about 10 times the nuclear density $\rho_{n}=2 \times 10^{14} \mathrm{~g} / \mathrm{cm}^{3}$. The vacuum boundary of the initial matter distribution is defined by the equation $p=0$, a condition corresponding to a surface density $\rho_{s}$ $=\rho_{0} e^{-1 / 4} \approx 0.778 \times 10^{14} \mathrm{~g} / \mathrm{cm}^{3}$, two times smaller in magnitude as the nuclear density. This condition also defines the physical radius of the initial matter distribution and defines a boundary for the null fluid.

Hence, as a possible physical model to describe highdensity matter in the final stages of the gravitational collapse we shall adopt the Hagedorn equation of state. In the following section we present the general solution of the gravitational field equations for the null Hagedorn fluid.

\section{SPHERICAL COLLAPSE OF THE HAGEDORN NULL FLUID}

From Eq. (11) we immediately obtain

$$
\frac{\rho(u, r)}{\rho_{0}}=\frac{1}{\rho_{0} r^{2}}\left[2 \frac{\partial m(u, r)}{\partial r}-\frac{q^{2}(u)}{r^{2}}\right] .
$$

With the use of the Hagedorn equation of state, Eq. (14), and of Eq. (15), Eq. (12), can be written in the form

$$
\begin{aligned}
-\frac{1}{r}\left[\frac{\partial^{2} m(u, r)}{\partial r^{2}}+\frac{q^{2}(u)}{r^{3}}\right]= & p_{0}+\rho_{0} \ln \left\{\frac { 1 } { \rho _ { 0 } r ^ { 2 } } \left[2 \frac{\partial m(u, r)}{\partial r}\right.\right. \\
& \left.\left.-\frac{q^{2}(u)}{r^{2}}\right]\right\} .
\end{aligned}
$$

By introducing a new variable

$$
w(u, r)=\ln \frac{\rho}{\rho_{0}}=\ln \left\{\frac{1}{\rho_{0} r^{2}}\left[2 \frac{\partial m(u, r)}{\partial r}-\frac{q^{2}(u)}{r^{2}}\right]\right\},
$$

it is easy to show that

$$
\frac{1}{\rho_{0} r}\left[\frac{\partial^{2} m(u, r)}{\partial r^{2}}+\frac{q^{2}(u)}{r^{3}}\right]=e^{w}\left(\frac{1}{2} r \frac{\partial w}{\partial r}+1\right) .
$$

Therefore Eq. (16) becomes

$$
r \frac{\partial w}{\partial r}=-2 \frac{\alpha+w+e^{w}}{e^{w}}
$$

where $\alpha=p_{0} / \rho_{0} \approx 0.25$. By integrating Eq. (19) we obtain

$$
\frac{r}{C(u)}=\exp \left(-\frac{1}{2} \int \frac{e^{w}}{\alpha+w+e^{w}} d w\right)=\exp \left[-\frac{1}{2} F(w)\right],
$$

where $C(u)$ is an arbitrary integration function and we denoted $F(w)=\int\left[e^{w} /\left(\alpha+w+e^{w}\right)\right] d w$. Equation (20) formally defines $w$ as a function of $\eta=r / C(u), w=H(\eta)$. The variation of the mass function is described by the equation

$$
2 \frac{\partial m(u, r)}{\partial r}=\frac{q^{2}(u)}{r^{2}}+\rho_{0} r^{2} e^{w}
$$

having the general solution given by

$$
\begin{aligned}
2 m(u, r)= & D(u)-\frac{q^{2}(u)}{C(u)} e^{(1 / 2) F(w)} \\
& -\frac{\rho_{0}}{2} C^{3}(u) \int \frac{\exp \left[2 w-\frac{3}{2} F(w)\right]}{\alpha+w+e^{w}} d w,
\end{aligned}
$$

where $D(u)$ is an arbitrary integration function. In the following we shall also denote:

$$
K(w)=\int \frac{\exp \left[2 w-\frac{3}{2} F(w)\right]}{\alpha+w+e^{w}} d w .
$$

The density and the pressure of the collapsing null Hagedorn fluid are given by 


$$
\rho(w)=\rho_{0} \exp (w), \quad p(w)=p_{0}+\rho_{0} w .
$$

The energy density $\mu$ of the radiation moving along the $u=$ const null hypersurfaces is given by

$$
\begin{aligned}
\mu(u, r)= & \frac{\dot{D}(u)}{C^{2}(u)} e^{F(w)}-\frac{2 q(u) \dot{q}(u)}{C^{3}(u)} e^{(3 / 2) F(w)} \\
& -\frac{3 \rho_{0}}{2} \dot{C}(u) e^{F(w)} K(w)+\frac{\rho_{0}}{2} \dot{C}(u) \frac{e^{2 w-F(w)}}{\alpha+w+e^{w}} \frac{d H}{d \eta}
\end{aligned}
$$

where a dot denotes the derivative with respect to $u$.

The function $r / C(u)$ can be represented as a power series of the parameter $w$ in the form

$$
\frac{r}{C(u)} \approx 1-\frac{2 w}{5}+\frac{w^{2}}{5}-\frac{w^{3}}{5}+\frac{13 w^{4}}{60}-\frac{367 w^{5}}{1500}+O^{6}[w],
$$

while the function $K(w)$ has the power series representation

$$
K(w) \approx \frac{4 w}{5}-\frac{8 w^{2}}{25}+\frac{52 w^{3}}{25}-\frac{59 w^{4}}{125}+\frac{351 w^{5}}{625}+O^{6}[w] .
$$

In the limit of large densities $\rho \rightarrow \infty$, which also implies $w \rightarrow \infty$, Eq. (19) becomes

$$
r \frac{\partial w}{\partial r} \approx-2
$$

with the general solution given by

$$
w(u, r) \approx \ln \frac{C(u)}{r^{2}} .
$$

Therefore in the asymptotic limit of very high densities, corresponding to $r \rightarrow 0$, the gravitational collapse of the Hagedorn null fluid is described by the equations

$$
\begin{aligned}
2 m(u, r) & \approx D(u)+\rho_{0} C(u) r-\frac{q^{2}(u)}{r}, \\
\rho(u, r) & \approx \rho_{0} \frac{C(u)}{r^{2}}, \quad p(u, r) \approx p_{0}+\rho_{0} \ln \frac{C(u)}{r^{2}}, \\
\mu(u, r) & \approx \frac{\dot{D}}{r^{2}}+\rho_{0} \frac{\dot{C}}{r}-\frac{2 q \dot{q}}{r^{3}} .
\end{aligned}
$$

In order to find the behavior of the solution in the opposite limit of large, but finite $r$, we note first that the boundary of the Hagedorn type matter distribution is defined by the equation $p=0$, corresponding to a value of the parameter $w=w_{s}=-p_{0} / \rho_{0} \approx-1 / 4$. Values of $w<w_{s}$ lead to the unphysical situation of negative pressure matter distribution, $p<0$ for $w<w_{s}$. In order to find the behavior of the solution near $w_{s}$, we represent $w(u, r)$ in the form $w(u, r)=w_{s}$ $+w_{1}(u, r)$, with $w_{1}(u, r)$ a small perturbation satisfying the condition $\left|w_{1}(u, r)\right| \ll\left|w_{s}\right|, \forall u, r$. Substituting into Eq. (19) gives

$$
r \frac{\partial w_{1}}{\partial r} \approx-2 \frac{w_{1}+e^{w_{s}} e^{w_{1}}}{e^{w_{s}} e^{w_{1}}} \approx-2 \frac{(1+s) w_{1}+s}{s\left(1+w_{1}\right)},
$$

where we denoted $s=\exp \left(w_{s}\right) \approx \exp (-1 / 4) \approx 0.778$. Hence for large $r$ the solution of Eq. (33) is given by

$$
\begin{aligned}
& \frac{s}{1+s} w_{1}+\frac{s \ln s}{(1+s)^{2}}+\frac{s}{(1+s)^{2}} \ln \left[1+\frac{1+s}{s} w_{1}\right]=-2 \ln r \\
& +C^{\prime}(u),
\end{aligned}
$$

where $C^{\prime}(u)$ is an arbitrary integration function. Since we have assumed that $w_{1}(u, r)$ is small, we can neglect in Eq. (34) the term containing the logarithmic function. Hence near the vacuum boundary of the high density Hagedorn fluid distribution we obtain

$$
w \approx w_{0}+\ln \frac{C(u)}{r^{2 k}}
$$

where $w_{0}=w_{s} s /(1+s) \approx-0.10, k=(1+s) / s=2.284$, and $C(u)$ is an arbitrary, $u$-dependent integration function.

Therefore in the limit $r \rightarrow \infty$ the general solution of the gravitational field equations can be approximated by

$2 m(u, r) \approx D(u)-\frac{q^{2}(u)}{r}+\frac{\rho_{0} e^{w_{0}}}{3-2 k} C(u) r^{3-2 k}$

$$
\approx D(u)-\frac{q^{2}(u)}{r}-0.6 \rho_{0} \frac{C(u)}{r^{1.56}},
$$

$$
\rho(u, r) \approx \rho_{0} e^{w_{0}} \frac{C(u)}{r^{2 k}} \approx 0.9 \rho_{0} \frac{C(u)}{r^{4.56}},
$$

$$
p(u, r) \approx p_{0}+\rho_{0} w_{0}+\rho_{0} \ln \frac{C(u)}{r^{2 k}} \approx \rho_{0}\left(0.15+\ln \frac{C(u)}{r^{4.56}}\right)
$$

$$
\begin{aligned}
\mu(u, r) & \approx 2 \frac{\dot{D}(u)}{r^{2}}-\frac{2 q(u) \dot{q}(u)}{r^{3}}+\frac{2 \rho_{0} e^{w_{0}}}{3-2 k} \dot{C}(u) r^{1-2 k} \\
& \approx 2 \frac{\dot{D}(u)}{r^{2}}-\frac{2 q(u) \dot{q}(u)}{r^{3}}-0.58 \rho_{0} \frac{\dot{C}(u)}{r^{3.56}}
\end{aligned}
$$

where $D(u)$ is an arbitrary integration function.

The variation of the ratio $r / C(u)$ as a function of $w$ is represented, by using Eq. (20), in Fig. 1.

As one can see from the figure, in the limit $w \rightarrow w_{s}=$ -0.25 , the ratio $r / C(u)$ tends to 1 , while for large $w, w$ $\rightarrow \infty$, that is, in the limit of very high densities, $r / C(u)$ tends to zero. Values of $w<w_{s}=-0.25$ lead to the violation of the 


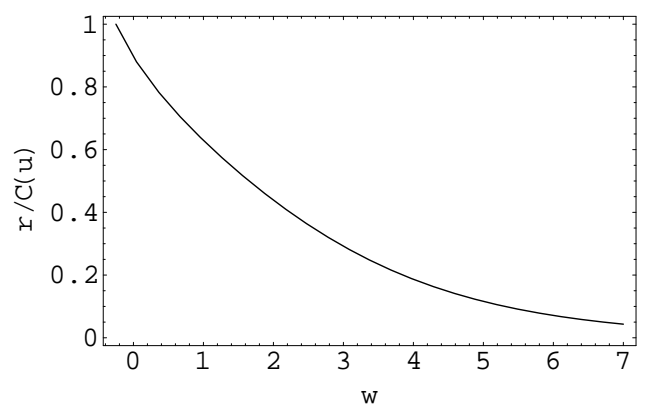

FIG. 1. Variation of the ratio $r / C(u)$ as a function of the parameter $w$.

dominant energy condition $p \geqslant 0$. The function $K(w)$ is represented, as a function of $r / C(u)$, in Fig. 2.

For the sake of comparison we shall also present the solution of the gravitational field equations corresponding to the collapse of the charged null Zeldovich fluid with $\rho=p$. In this case Eqs. (11) and (12) give immediately

$$
\frac{2}{r^{2}} \frac{\partial m(u, r)}{\partial r}+\frac{1}{r} \frac{\partial^{2} m(u, r)}{\partial r^{2}}=0,
$$

with the general solution given by

$$
m(u, r)=B(u)-\frac{A^{2}(u)}{r},
$$

where $A(u)$ and $B(u)$ are arbitrary integration functions.

The other physical quantities characterizing the collapsing Zeldovich fluid are given by

$$
\begin{aligned}
& \rho(u, r)=p(u, r)=\frac{2 A^{2}(u)-q^{2}(u)}{r^{4}}, \\
& \mu(u, r)=\frac{2}{r^{2}}\left(\frac{d B}{d u}-\frac{2}{r} A(u) \frac{d A}{d u}\right) .
\end{aligned}
$$

In all these cases the electromagnetic current follows from the Maxwell equation (7) and can be generally represented as

$$
j^{\mu}=\frac{1}{4 \pi r^{2}} \frac{d q(u)}{d u} l^{\mu}
$$

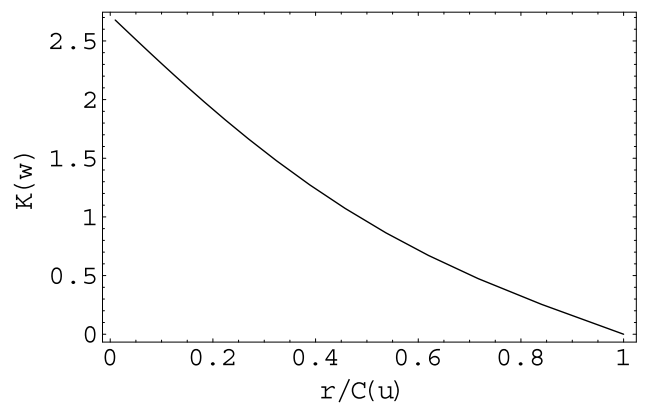

FIG. 2. Variation of $K(w)$ as a function of $r / C(u)$.
The energy-momentum tensor of the mixture of fluids under consideration belongs to the type-II fluids [21]. The energy conditions are the weak, strong, and dominant energy conditions $\mu \geqslant 0, \rho \geqslant 0, p \geqslant 0, \rho \geqslant p \geqslant 0$ and can be satisfied for both Hagedorn and Zeldovich fluids by appropriately choosing the arbitrary functions $A(u), B(u), C(u)$, and $D(u)$ that characterize the injection and initial distribution of the mass and $q(u)$ that describes the variation of the charge. For the Hagedorn fluid the conditions $\rho \geqslant 0$ and $p \geqslant 0$ are trivially satisfied for the values of the parameter $w$ in the range $w \in\left(w_{s}, \infty\right)$, corresponding to the range of densities of $\rho \in\left(\rho_{s}, \infty\right)$, with $w_{s}=-0.25$ and $\rho_{s}=0.78 \times 10^{14} \mathrm{~g} / \mathrm{cm}^{3}$. Due to the choice of the parameters in the equation of state the dominant energy condition $\rho \geqslant p$ is also satisfied. The condition $\mu \geqslant 0$ is equivalent to $d m / d u \geqslant 0$, and in the approximation of high densities leads to

$$
\frac{d D(u)}{d u}+\rho_{0} \frac{d C(u)}{d u} r \geqslant 2 q(u) \frac{d q(u)}{d u} \frac{1}{r},
$$

imposing a simultaneous constraint on all three functions $C(u), D(u)$, and $q(u)$. For small values of $r$ and for a charged collapsing high density fluid, the right-hand side of Eq. (45) dominates and this energy condition could not hold. One possibility to satisfy Eq. (45) for all $r$ is to assume that the function $q(u)$ behaves so that $d q^{2}(u) / d u \rightarrow 0$ for $r \rightarrow 0$. This means that the charge in the singular point $r=0$ is constant for all times. Alternatively, we may suppose that at extremely small radii matter is generated so as to satisfy the energy condition. For neutral $q \equiv 0$ matter Eq. (45) is easily satisfied by choosing $d C(u) / d u>0$ and $d D(u) / d u>0$. In the case of the Zeldovich fluid the energy conditions are satisfied by choosing the functions $A, B$, and $q$ so that $A^{2}(u) \geqslant q^{2}(u) / 2$ and $\dot{B}(u) \geqslant 2 A(u) \dot{A}(u) / r$.

The radii of the apparent horizon of the metric (1) are given by the solution of the equation $2 m=r$. If $\lim _{u \rightarrow \infty} C(u)=C_{0}=$ const, $\quad \lim _{u \rightarrow \infty} D(u)=D_{0}=$ const and $\lim _{u \rightarrow \infty} q(u)=q_{0}=$ const, then the algebraic equation determining the radii of the apparent horizons in the case of the Hagedorn fluid is

$$
C_{0} e^{-(1 / 2) F(w)}+\frac{\rho_{0}}{2} C_{0}^{3} K(w)+\frac{q_{0}^{2}}{C_{0}} e^{(1 / 2) F(w)}=D_{0},
$$

which in general may have multiple solutions.

In the case of the collapse of the Zeldovich fluid the radii of the apparent horizon are given by the solutions of the equation

$$
2 B_{0}-2 \frac{A_{0}^{2}}{r}=r
$$

where $A_{0}=\lim _{u \rightarrow \infty} C(u)=$ const and $B_{0}=\lim _{u \rightarrow \infty} D(u)$ $=$ const. The radii of the apparent horizon are

$$
r_{1,2}=B_{0} \pm \sqrt{B_{0}^{2}-2 A_{0}^{2}} \text {. }
$$

The singularities of the matter filled Vaidya space-time can be recognized from the behavior of the energy density 
and curvature scalars like, e.g., $R_{\alpha \beta} R^{\alpha \beta}$ and $R_{\alpha \beta \gamma \delta} R^{\alpha \beta \gamma \delta}$, given in the high density limit for the collapsing Hagedorn fluid by

$$
\begin{aligned}
R_{\alpha \beta} R^{\alpha \beta} & =\frac{2}{r^{8}}\left[r^{4} \rho_{0}^{2} C^{2}(u)+2 r^{2} \rho_{0} C(u) q^{2}(u)+2 q^{4}(u)\right], \\
R_{\alpha \beta \gamma \delta} R^{\alpha \beta \gamma \delta} & =4 \frac{r^{4} \rho_{0}^{2} C^{2}(u)-2 r^{3} \rho_{0} C(u) D(u)+3 r^{2} D^{2}(u)-2 r^{2} \rho_{0} C(u) q^{2}(u)+12 r D(u) q^{2}(u)+14 q^{4}(u)}{r^{8}},
\end{aligned}
$$

and which diverge for $r \rightarrow 0$.

\section{OUTGOING RADIAL NULL GEODESICS EQUATION}

The central shell-focussing singularity (i.e., that occurring at $r=0$ ) is naked if the radial null-geodesic equation admits one or more positive real roots $X_{0}$ [2]. In the case of the pure Vaidya space-time it has been shown that for a linear mass function $2 m(u)=\lambda u$ the singularity at $r=0, u=0$ is naked for $\lambda \leqslant \frac{1}{8}$ [42]. Hence it is important to investigate whether the gravitational collapse of high density matter described by the Hagedorn and Zeldovich equations of state could result in the formation of naked singularities.

We consider first the case of the gravitational collapse of the Hagedorn fluid. In order to simplify the calculations we chose some simple particular expressions for the functions $C(u), D(u)$, and $q(u)$, e.g., $C(u)=\alpha_{0} u, D(u)=\beta_{0} u$, and $q(u)=q_{0} u$, with $\alpha_{0}>0, \beta_{0}>0$ and $q_{0} \geqslant 0$ const. With this choice the equation of the radially outgoing, future-directed null geodesic originating at the singularity can be written as

$$
\frac{d u}{d r}=\frac{2}{1-\beta_{0}\left(\frac{u}{r}\right)+q_{0}^{2}\left(\frac{u}{r}\right)^{2}+\frac{\rho_{0} \alpha_{0}^{3}}{2} u^{2}\left(\frac{u}{r}\right) K(w)} .
$$

For the geodesic tangent to be uniquely defined and to exist at the singular point $r=0, u=0$ of Eq. (51) the following condition must hold [2]:

$$
\lim _{u, r \rightarrow 0} \frac{u}{r}=\lim _{u, r \rightarrow 0} \frac{d u}{d r}=X_{0}
$$

When the limit exists and $X_{0}$ is real and positive, there is a future directed, non-space-like geodesic originating from $r=0, u=0$. In this case the singularity will be, at least, locally naked.

Since the function $K(w)$ is finite for all $w=H(r / C(u))$, and we assume that $\lim _{u, r \rightarrow 0} u / r$ is also finite, it follows that $\lim _{u, r \rightarrow 0} u^{2}(u / r) K(w)=0$. Therefore it follows that for the null geodesic Eq. (51) condition (52) leads to the following third order algebraic equation:

$$
q_{0}^{2} X_{0}^{3}-\beta_{0} X_{0}^{2}+X_{0}-2=0 .
$$

In the case of a neutral Hagedorn fluid with $q(u) \equiv 0$, Eq. (53) reduces to a second order algebraic equation with two roots, $X_{01,2}=\left(1 \pm \sqrt{1-8 \beta_{0}}\right) / 2 \beta_{0}$. Therefore the condition for the formation of a naked singularity is $\beta_{0}<1 / 8$. For $\beta_{0}$ $>1 / 8$, as a result of the collapse of the Hagedorn type fluid, a black hole is formed.

For $q(u) \neq 0$ the condition of the existence of at least one real solution of Eq. (53) is

$$
108 q_{0}^{4}+4 q_{0}^{2}+8 \beta_{0}^{3} \geqslant \beta_{0}^{2}+36 \beta_{0} q_{0}^{2} .
$$

Therefore, by appropriately choosing the constants $q_{0}$ and $\beta_{0}$, it is always possible to construct a positive solution of Eq. (53).

In the case of the collapse of the Zeldovich fluid and by assuming for the arbitrary $u$-dependent integration functions the form $A(u)=a_{0} u$ and $B(u)=b_{0} u$, with $a_{0}, b_{0}$ nonnegative constants, the equation of the radially outgoing, future-directed null geodesic originating at the singularity is

$$
\frac{d u}{d r}=\frac{2}{1-b_{0}\left(\frac{u}{r}\right)+a_{0}^{2}\left(\frac{u}{r}\right)^{2}} .
$$

The algebraic condition for the formation of a naked singularity is given by the requirement that the equation,

$$
a_{0}^{2} X_{0}^{3}-b_{0} X_{0}^{2}+X_{0}-2=0,
$$

has at least one positive root $X_{0}>0$. The condition that the above equation has at least one real root can be written as

$$
\frac{a_{0}^{2}}{b_{0}^{2}} \geqslant \frac{1-8 b_{0}}{108 a_{0}^{2}-36 b_{0}+4} .
$$

This condition can be satisfied, for example, by choosing $b_{0}<1 / 8$ and $a_{0}>1 / \sqrt{216}$. Therefore, as in the case of the charged Hagedorn fluid, it is always possible to construct a positive solution of Eq. (56).

\section{COLLAPSING HAGEDORN MATTER-A POSSIBLE SOURCE OF GAMMA-RAY BURSTS}

Gamma-ray bursts (GRBs) are cosmic gamma ray emissions with typical fluxes of the order of $10^{-5}$ to 5 $\times 10^{-4} \mathrm{erg} \mathrm{cm}^{-2}$ with the rise time as low as $10^{-4} \mathrm{~s}$ and the duration of bursts from $10^{-2}$ to $10^{3} \mathrm{~s}$ [43]. The distribution of the bursts is isotropic and they are believed to have a cosmological origin, recent observations suggesting that 
GRBs might originate at extra-galactic distances [43]. The large inferred distances imply isotropic energy losses as large as $3 \times 10^{53} \mathrm{erg}$ for GRB 971214 and $3.4 \times 10^{54} \mathrm{erg}$ for GRB 990123 [44]. In the present section we shall use CGS units. The widely accepted interpretation of the phenomenology of $\gamma$-ray bursts is that the observable effects are due to the dissipation of the kinetic energy of a relativistically expanding fireball, whose primal cause is not yet known [43].

The proposed models for the energy source involve the merger of binary neutron stars [45], the capture of neutron stars by black holes [46], differentially rotating neutron stars [47], or neutron star-quark star conversion [48], etc. However, the most popular model involves the violent formation of an approximately one solar mass black hole, surrounded by a similarly massive debris torus. The formation of the black hole and debris torus may take place through the coalescence of a compact binary or the collapse of a quickly rotating massive stellar core [49]. There are still many open problems concerning GRBs, from which the most important is the problem of the source of the large energy emission during the bursts. On the other hand, naked singularities as sources of $\gamma$-ray bursts have also been proposed [50-52]. The fact that explosive radiation can be emitted during the gravitational collapse to a naked singularity of a dust ball has also been pointed out recently by [53].

As an astrophysical application of the Hagedorn fluid collapse in the Vaidya geometry we consider the possibility that gamma-ray bursts could in fact be energy emission during the collapse of a neutral, $q \equiv 0$, high density matter in the Hagedorn phase, ending with the formation of a naked singularity. An estimation of the energy emitted during the collapse shows that it is of the same order of magnitude as the one measured during $\gamma$-ray bursts. Hence this mechanism could provide a valuable explanation for this phenomenon, also opening the possibility of the observational investigation of the astrophysical properties of the naked singularities.

The arbitrary integration functions $C(u)$ and $D(u)$ appearing in Eq. (22) describe the injection and initial distribution of the mass, respectively. Their exact mathematical form cannot be obtained in the framework of the present formalism. The only requirements that the functional form of these functions must satisfy are the dominant energy conditions and the condition that the equation of the radially outgoing, future-directed null geodesics, originating at the singularity $r=0$, be uniquely defined and exist at the singular point $r$ $=0$ and has at least one positive root. A positive root of the geodesic equation leads to the possibility of formation of a naked singularity as a result of the collapse. Some simple integration functions satisfying the condition on the geodesic equation, as well as the dominant energy conditions, are, for example, $C(u)=\alpha_{0} u, D(u)=\beta_{0} u$, with $\alpha_{0}>0, \beta_{0}>0$ constants. With this choice a naked singularity may form during the collapse of the Hagedorn fluid if and only if the algebraic equation $\beta_{0} X_{0}^{2}-X_{0}+2=0$ has at least one positive root $X_{0}$ $>0$. This condition requires $\beta_{0}<1 / 8$. Of course many other choices are also possible.

Naked singularities are gravitational singularities that are not covered by a horizon. Near the singularity the space-time curvature and the gravitational tidal forces grow very strongly. During the collapse naked singularities could emit powerful bursts of radiation visible to a distant external observer situated far away from the sight of the collapse [50].

As a first physical parameter we need to estimate is the time $t_{f f}$ necessary for a matter element at the surface of the star to reach the center $r=0$ of the collapsed object, as a function of the mass at the center. This can be done by evaluating the time derivative of the mass, given by Eq. (30) with $q=0$, at the center of the naked singularity:

$$
\left.\frac{d m}{d t}\right|_{r=0}=\frac{c^{3}}{2 G}\left(\frac{d D(u)}{d u}\right)_{r=0} .
$$

By integrating the above equation from $t=0$ to $t_{f f}$ we obtain

$$
\left.M\right|_{r=0}=\frac{c^{3}}{2 G} \int_{0}^{t_{f f}}\left(\frac{d D(u)}{d u}\right)_{r=0} d t,
$$

where we assumed $\left.m(0)\right|_{r=0}=0$ and denoted $\left.m\left(t_{f f}\right)\right|_{r=0}$ $=\left.M\right|_{r=0}=0$. In order to find an explicit expression for $t_{f f}$ we shall approximate the integral in Eq. (59) by using the first mean value theorem for integrals, which states that for any arbitrary function $f(t), \int_{a}^{b} f(t) d t=(b-a) f(c)$, where $a<c<b . f(c)$ is called the average value of $f$. Hence, in the following we shall approximate the derivatives of the arbitrary the arbitrary integration functions and the functions themselves by their average values. Therefore from Eq. (59) we obtain

$$
t_{f f}=\left.\frac{2 G}{c^{3} \beta_{0}} M\right|_{r=0},
$$

where we denoted by $\beta_{0} \geqslant 0$ the average value of the function $[d D(u) / d u]_{r=0}, \beta_{0}=[d D(t) / d(t)]_{t=\theta}$, where $0<\theta$ $<t_{\text {eff }}$.

The initial mass distribution of the Hagedorn fluid can be obtained from the study of the mass distribution at the initial moment $t=0$ :

$$
\left.\frac{d m}{d r}\right|_{t=0}=\frac{c^{2}}{2 G}\left[\left.\frac{d D(u)}{d u}\right|_{t=0}+\left.\rho_{0} r \frac{d C(u)}{d u}\right|_{t=0}\right] .
$$

By integrating this equation, and using again the first mean value theorem, it follows that the initial mass profile of the Hagedorn fluid can be represented as

$$
\left.m(r)\right|_{t=0}=\frac{c^{2}}{2 G} \alpha_{0} r,
$$

where $\alpha_{0} \geqslant 0$ is the average value of the $r$-dependent function $h(r)=\left.\left[d D(u) / d u+\rho_{0} r(d C(u) / d u)\right]\right|_{t=0}$, with the function $h(r)$ estimated at some point $r=\sigma, 0<\sigma<R$, with $R$ the radius of the Hagedorn fluid distribution. Hence $\alpha_{0}$ $=h(\sigma)$. From this equation we can see that our approach implies a linear profile of the initial mass distribution of the star. $\alpha_{0}$, the average value of the spatial derivatives of the integration functions $D(u)$ and $C(u)$ at $t=0$, is completely 
determined by the initial conditions, which is by the total initial mass of the $\left.\operatorname{star} M\right|_{t=0}$ and by its initial radius $R$ via the relation

$$
\alpha_{0}=\frac{\left.2 G M\right|_{t=0}}{c^{2} R} .
$$

In order to calculate the energy emitted during the Hagedorn fluid collapse we shall admit that the luminosity of the collapsing object should not exceed the rate of collapsing matter energy [52]. The variation of the mass of the high density fluid during collapse is given by

$$
\begin{aligned}
\Delta \frac{2 G m(u, r)}{c^{2}} & =\left[\Delta D(u)+\rho_{0} r \Delta C(u)+\rho_{0} C(u) \Delta r\right] \\
& \approx\left[\frac{d D(u)}{d u} \Delta u+\rho_{0} r \frac{d C(u)}{d u} \Delta u+\rho_{0} C(u) \Delta r\right] .
\end{aligned}
$$

The variation of the advanced time coordinate $u$ is given by the approximate expression

$$
\Delta u=c \Delta t+\Delta r=c \Delta t\left(1+\frac{1}{c} \frac{\Delta r}{\Delta t}\right) \approx c \Delta t\left(1+\frac{v_{f}}{c}\right),
$$

where we defined $v_{f}=\Delta r / \Delta t$ as being the speed of the collapsing fluid as measured by a local observer. Then we obtain

$$
\begin{aligned}
\Delta \frac{2 G m(u, r)}{c^{2}} \approx & {\left[\frac{d D(u)}{d u}+\rho_{0} r \frac{d C(u)}{d u}+\rho_{0} C(u) \frac{v_{f}}{c}(1\right.} \\
& \left.\left.+\frac{v_{f}}{c}\right)^{-1}\right] c\left(1+\frac{v_{f}}{c}\right) \Delta t .
\end{aligned}
$$

We assume that the energy emission occurs mainly from a small region near the center of the naked singularity. Hence we shall evaluate Eq. (65) near $r$ very close to zero. Then from Eq. (58) we can roughly approximate $d D(u) / d u$ $\approx\left(2 G / c^{3}\right)(d m / d t) \approx 2 G / c^{3}\left(M / t_{f f}\right)=\beta_{0}$. Since for a small $r, C(c t+r) \approx C(c t)$, we can also neglect in this limit the term $\rho_{0} r[d C(u) / d u]$. From the field Eq. (11) and from Eq. (24) it immediately follows that $2[\partial m(u, r] / \partial r)$ $=\left(8 \pi G / c^{2}\right) r^{2} \rho \approx \rho_{0} C(u)$. Taking into account Eq. (62) we immediately find $\rho_{0} C(u) \approx 4 \pi \alpha_{0} \approx\left(8 \pi G M / c^{2} R\right)$. The velocity $v_{f}$ of the collapsing matter can be obtained from $v_{f}$ $\approx R / t_{f f} \approx\left(c^{3} R \beta_{0}\right) /\left(\left.2 G M\right|_{r=0}\right)$.

With the use of the previous results we obtain for the total energy emitted per unit time during the collapse of a Hagedorn fluid to a naked singularity the expression:

$$
\frac{\Delta E_{r}}{\Delta t} \approx \frac{1}{2}\left[\beta_{0}+4 \pi \alpha_{0} \frac{v_{f}}{c}\left(1+\frac{v_{f}}{c}\right)^{-1}\right]\left(1+\frac{v_{f}}{c}\right) \frac{c^{5}}{G} \mathrm{erg} \mathrm{s}^{-1} .
$$

Hence we have expressed the total energy of the radiation, which could be emitted from a naked singularity, in terms of the average values of the derivatives of the arbitrary integration functions. By assuming an emission time of the order of $\Delta t \sim 10^{-4} \mathrm{~s}$ and a velocity of the collapsing matter of the order $v_{f}=6 \times 10^{8} \mathrm{~cm} / \mathrm{s}$ we obtain a value of the emitted energy $\quad \Delta E_{\gamma} \approx 1.85 \times\left\{\beta_{0}+4 \pi \alpha_{0}\left(v_{f} / c\right)\left[1+\left(v_{f} / c\right)\right]^{-1}\right\}$ $\times 10^{55} \mathrm{erg}$. Therefore the energy which could be emitted during the collapse of the Hagedorn fluid to a naked singularity could exceed in magnitude the energy $\Delta E_{\gamma} \approx 4$ $\times 10^{54} \mathrm{erg}$, observed for GRB990123 [44]. Of course the exact value of the energy depends on the exact numerical values of the constants $\alpha_{0}$ and $\beta_{0}$. By comparing Eq. (66) with the observational data it is possible to determine the values of $\alpha_{0}$ and $\beta_{0}$.

Under the effect of the collapse the compact object will heat up to a temperature of the order of $T \sim T_{H} \sim 10^{13} \mathrm{~K}$, higher than that occurring in supernova explosions. Since $T_{H}$ cannot be exceeded, the gravitational energy of the compact object is converted into new particles, which will be generated during the collapse. Most of the newly created particle will decay via weak interaction processes. As a result a neutrinosphere will form around the naked singularity. Therefore the main energy loss mechanism of the super-heated collapsed astrophysical object would be neutrino radiation. The neutrinos and antineutrinos interact with protons and neutrons via the URCA processes $n+\nu_{e} \rightarrow p+e^{-}, p+\bar{\nu}_{e} \rightarrow n$ $+e^{+}$. At temperatures higher than the nuclear Fermi temperature $k T^{F, N}=\left(6 \pi^{2} / g\right)^{2 / 3}(\hbar / 2 m)(N / V)^{2 / 3}$, which can also be expressed in the form $T_{11}^{F, N}=1.47 \times 10^{-3} \rho_{9}^{2 / 3}$ [49], the integrated optical neutrino depth is unity. Hence the deposition energy can be estimated as $E \approx\left(1-e^{-\tau}\right) \Delta E_{\gamma}$ ergs. The process $\gamma+\gamma \rightarrow e^{+}+e^{-}$will generate a fireball that will expand outward. The expanding shell interacts with the interstellar medium surrounding the Hagedorn type naked singularity, and the kinetic energy is finally radiated through nonthermal processes in shocks [48].

The energy released during the collapse of the Hagedorn and radiation fluids into a naked singularity has the same order of magnitude as that observed in the case of gamma ray bursts. This strongly suggests the possibility that gamma ray bursts could be massive compact objects, formed from a Hagedorn fluid, collapsing to a naked singularity in a cosmological environment.

\section{CONCLUSIONS}

In the present paper we have considered the collapse of a Hagedorn type fluid in the Vaidya geometry. The exact solution obtained represents the generalization for the Hagedorn type matter of the collapsing solutions previously obtained by Vaidya [3], Bonnor and Vaidya [6], Lake and Zannias [7], and Husain [21]. From a mathematical point of view the solution is represented in a parametric form. It satisfies all the energy conditions and consequently describes the collapse to a singular state. The possible occurrence of a central naked singularity has also been investigated and it has been shown that, at least for a particular choice of the parameters, a naked singularity is formed. Depending on the initial distribution of density and velocity and on the constitutive nature of the collapsing matter, either a black hole or a naked 
singularity is formed. The values of the parameters in the solution (22) and (24) determine which of these possibilities occurs. The solution describing the collapse of both the Hagedorn and Zeldovich matter is asymptotically flat, but this condition does not play any significant role in the formation of the naked singularity.

The Hagedorn type matter may reside as a permanent component of neutron stars core at high temperatures or densities and form stable compact stellar objects. In fact, from a physical point of view, it seems that the high density limit for the equation of state described by the Hagedorn equation of state is one of the best and more realistic candidates for the study of properties of collapsing objects. It also serves to illustrate the much richer interplay that can occur among particle physics and general relativity, when more involved quantum field theoretical models are considered.

As a possible simple astrophysical application of this collapsing solution we have considered the possibility that gamma ray bursts could be energy emission during the collapse of a high density star, ending in the formation of a naked singularity. The radiated energy during this process could be as high as $10^{55} \mathrm{ergs}$. Thus the naked singularity explosion could be a candidate for the central engine of a gamma ray burst.
[1] R. Penrose, Nuovo Cimento 1, 252 (1969).

[2] P.S. Joshi, Global Aspects in Gravitation and Cosmology (Clarendon, Oxford, 1993).

[3] P.C. Vaidya, Proc.-Indian Acad. Sci., Sect. A 33, 264 (1951).

[4] R.C. Tolman, Proc. Natl. Acad. Sci. U.S.A. 20, 169 (1934).

[5] J.P.S. Lemos, Phys. Rev. Lett. 68, 1447 (1992).

[6] W.B. Bonnor and P.C. Vaidya, Gen. Relativ. Gravit. 2, 127 (1970).

[7] K. Lake and T. Zannias, Phys. Rev. D 43, 1798 (1991).

[8] B.T. Sullivan and W. Israel, Phys. Lett. 79A, 371 (1980).

[9] E. Poisson and W. Israel, Phys. Rev. D 41, 1796 (1990).

[10] A. Ori, Phys. Rev. D 57, 4745 (1998).

[11] T.P. Singh and P.S. Joshi, Class. Quantum Grav. 13, 559 (1996).

[12] S. Jhingan, P.S. Joshi, and T.P. Singh, Class. Quantum Grav. 13, 3057 (1996).

[13] P.S. Joshi and A. Krolak, Class. Quantum Grav. 13, 3069 (1996).

[14] J.H. Dwivedi and P.S. Joshi, Class. Quantum Grav. 14, 1223 (1997).

[15] T.P. Singh, Phys. Rev. D 58, 024004 (1998).

[16] S. Barve and T.P. Singh, Mod. Phys. Lett. A 12, 2415 (1997).

[17] L.M. Gonzalez-Romero, Class. Quantum Grav. 15, L59 (1998).

[18] M.K. Parikh and F. Wilczek, Phys. Lett. B 449, 24 (1999).

[19] B.C. Nolan, Phys. Rev. D 60, 024014 (1999).

[20] A. Ishibashi and A. Hosoya, Phys. Rev. D 60, 104028 (1999).

[21] V. Husain, Phys. Rev. D 53, R1759 (1996).

[22] A. Wang and Y. Wu, Gen. Relativ. Gravit. 31, 107 (1999).

[23] J.F. Villas da Rocha, Int. J. Mod. Phys. D 11, 113 (2002).

[24] E.N. Glass and J.P. Krisch, Phys. Rev. D 57, 5945 (1998).

[25] E.N. Glass and J.P. Krisch, Class. Quantum Grav. 16, 1175 (1999).

[26] K.S. Govinder and M. Govender, Phys. Rev. D 68, 024034 (2003).

[27] T. Harko and K.S. Cheng, Phys. Lett. A 266, 249 (2000).

[28] S.G. Ghosh and N. Dadhich, Phys. Rev. D 65, 127502 (2002).
[29] S.G. Ghosh and N. Dadhich, Gen. Relativ. Gravit. 35, 359 (2003).

[30] R. Hagedorn, Nuovo Cimento, Suppl. 3, 147 (1965).

[31] S.B. Giddings, Phys. Lett. B 226, 55 (1989).

[32] G. Grignani, M. Orselli, and G.W. Semenoff, J. High Energy Phys. 11, 058 (2001).

[33] M. Maggiore, Nucl. Phys. B525, 413 (1998).

[34] M. Bellini, Gen. Relativ. Gravit. 34, 1483 (2002).

[35] J. Magueijo and L. Pogosian, Phys. Rev. D 67, 043518 (2003).

[36] B.A. Bassett, M. Borunda, M. Serone, and S. Tsujikawa, Phys. Rev. D 67, 123506 (2003).

[37] L.D. Landau and E. Lifshitz, The Classical Theory of Fields (Butterworth-Heinemann, Oxford, 1975).

[38] S.L. Shapiro and S.A. Teukolsky, Black Holes, White Dwarfs and Neutron Stars (Wiley, New York, 1983).

[39] W. Broniowski and W. Florkowski, Phys. Lett. B 490, 223 (2000).

[40] R. Hagedorn, Astron. Astrophys. 5, 184 (1970).

[41] C.E. Rhoades, Jr. and R. Ruffini, Astrophys. J., Lett. Ed. 163, L83 (1971).

[42] I.H. Dwiwedi and P.S. Joshi, Class. Quantum Grav. 8, 1339 (1991).

[43] T. Piran, Phys. Rep. 333, 529 (1998).

[44] S.R. Kulkarni et al., Nature (London) 398, 389 (1999).

[45] T. Piran, Astrophys. J. Lett. 389, L45 (1992).

[46] B. Carter, Astrophys. J. Lett. 391, L67 (1992).

[47] W. Kluzniak and M. Ruderman, Astrophys. J. Lett. 505, L113 (1998).

[48] K.S. Cheng and Z.G. Dai, Phys. Rev. Lett. 77, 1210 (1996).

[49] P. Meszaros and M.J. Rees, Astrophys. J. 405, 278 (1993).

[50] S.K. Chakrabarti and P.S. Joshi, Int. J. Mod. Phys. D 3, 647 (1994).

[51] T.P. Singh, Gen. Relativ. Gravit. 30, 1563 (1998).

[52] T. Harko and K.S. Cheng, in Stellar Astrophyics, edited by K. S. Cheng et al. (Kluwer, Dordrecht, 2000), p. 139.

[53] T. Harada, H. Iguchi, and K. Nakao, Phys. Rev. D 61, 101502(R) (2000). 